\title{
Clinical Usefulness of the Korean Developmental Screening Test (K-DST) for Developmental Delays
}

\author{
Chul Hoon Jang, $\mathrm{MD}^{1}$, Seong Woo Kim, $\mathrm{MD}^{2}$, Ha Ra Jeon, $\mathrm{MD}^{2}$, \\ Da Wa Jung, $\mathrm{MD}^{2}$, Han Eol Cho, $\mathrm{MD}^{1}$, Jiyong Kim, $\mathrm{MD}^{3}$, Jang Woo Lee, $\mathrm{MD}^{2}$
}

\begin{abstract}
${ }^{1}$ Department of Rehabilitation Medicine and Rehabilitation Institute of Neuromuscular Disease, Yonsei University College of Medicine, Seoul; ${ }^{2}$ Department of Physical Medicine and Rehabilitation, National Health Insurance Service Ilsan Hospital, Goyang; ${ }^{3}$ Department of Physical Medicine and Rehabilitation, Inje University Ilsan Paik Hospital, Goyang, Korea
\end{abstract}

Objective To evaluate the clinical usefulness of the Korean Developmental Screening Test (K-DST) via comparison with Korean Ages and Stages Questionnaire (K-ASQ) for the diagnosis of developmental delay in pediatric patients. Methods The K-DST and K-ASQ were used to screen pediatric patients who visited the hospital for evaluation and diagnosis of delayed development. Korean Bayley Scales of Infant Development-II (K-BSID-II) or Korean Wechsler Preschool and Primary Scale of Intelligence III (K-WPPSI-III) were used for the standardized assessment. Moreover, the final clinical diagnosis was confirmed by three expert physicians (rehabilitation doctor, psychiatrist, and neurologist). The sensitivity and specificity of each screening tool for the final diagnosis were investigated and correlated with standardized assessments.

Results A total of 145 pediatric consultations were conducted, which included 123 developmental disorders (40 autism spectrum disorders, 46 global developmental delay/intellectual disability, and 37 developmental language disorders) and another 22 that were not associated with any such disorders. The sensitivity and specificity of K-DST based on the final clinical diagnosis were $82.9 \%$ and $90.9 \%$, respectively, which were not significantly different from that of K-ASQ (83.7\% and 77.3\%). Both K-DST and K-ASQ showed good correlation with K-BSID-II and K-WPPSI-III. No significant difference was found between the K-DST and K-ASQ measures.

Conclusion K-DST is an excellent screening tool and is expected to replace K-ASQ with high validity.

Keywords Developmental disabilities, Intellectual disability, Motor skills disorders, Autism spectrum disorder, Communication disorders

Received January 22, 2019; Accepted May 8, 2019

Corresponding author: Jang Woo Lee

Department of Physical Medicine and Rehabilitation, National Health Insurance Service Ilsan Hospital, 100 Ilsan-ro, Ilsandong-gu, Goyang 10444, Korea. Tel: +82-31-900-6965, Fax: +82-31-900-6967, E-mail: medipia29@nhimc.or.kr

ORCID: Chul Hoon Jang (http://orcid.org/0000-0001-9432-3473); Seong Woo Kim (http://orcid.org/0000-0002-1548-8147); Ha Ra Jeon (http://orcid. org/0000-0002-4234-8086); Da Wa Jung (http://orcid.org/0000-0002-2420-3553); Han Eol Cho (http://orcid.org/0000-0001-5625-3013); Jiyong Kim (http://orcid.org/0000-0003-4693-8400); Jang Woo Lee (http://orcid.org/0000-0002-2634-0375).

(a) This is an open-access article distributed under the terms of the Creative Commons Attribution Non-Commercial License (http://creativecommons.org/ licenses/by-nc/4.0) which permits unrestricted noncommercial use, distribution, and reproduction in any medium, provided the original work is properly cited. Copyright ( 2019 by Korean Academy of Rehabilitation Medicine 


\section{INTRODUCTION}

Developmental disability refers to decreased physical, psychological, cognitive, or linguistic abilities detected in early childhood. Developmental disability has a 5\%-10\% prevalence and is generally associated with conditions such as cerebral palsy, intellectual disability, learning disorder, attention deficit hyperactivity disorder, and autistic spectrum disorder (ASD) [1]. As infants and children should go through timely developmental milestones, early detection of developmental disabilities is critical for appropriate interventions [2,3].

The Korean Ages and Stages Questionnaire (K-ASQ) and Denver Developmental Screening Test II (DDST-II) were selected for use in the initial screening of developmental delays. However, the DDST-II is not appropriate in an outpatient clinic because of the need for a skilled examiner and extended duration. Although K-ASQ can be easily administered in an outpatient setting or via a phone interview, it is associated with several challenges because it was developed in a different cultural background and regardless of age $[4,5]$. Under sponsorship from the Ministry of Health and Welfare and the Korea Centers for Disease Control and Prevention, the Korean Developmental Screening Test (K-DST) for infants and children was developed between 2011 and 2014 by the Korean Society of Pediatric Rehabilitation and Developmental Medicine and the Korean Academy of Child and Adolescent Psychiatry [6]. K-DST is a screening tool completed by caregivers and is indicated for infants and children between the ages of 4 months and 71 months. Unlike K-ASQ, K-DST is not associated with age restrictions and is more appropriate for the Korean public, as it was developed and standardized for Korean infants and children. Although it was reported that K-DST has outstanding reliability and validity and clinical significance [6-8], it has never been directly compared with K-ASQ.

In this study, the caregiver reports involving K-ASQ and K-DST were compared to determine the relevance of KDST as a screening tool for developmental disabilities in the Korean pediatric population.

\section{MATERIALS AND METHODS}

\section{Study population}

From April 2010 to November 2011, outpatients who visited the Developmental Delay Clinic of National Health Insurance Service Ilsan Hospital for evaluation or diagnosis of delayed development were selected as participants. As K-DST can be used in infants and children between the ages of 4 months and 71 months, outpatients in this age range were selected to participate in this study.

\section{Study design}

The collected data of the retrospective study include KASQ, K-DST, the Korean Bayley Scales of Infant Development-II (K-BSID-II), and the Korean Wechsler Preschool and Primary Scale of Intelligence III (K-WPPSI-III). The caregivers of the participants were asked to fill out both K-DST and K-ASQ documents. K-BSID-II or K-WPPSI-III were used as standardized assessments in specific cases as needed and conducted by three skilled psychotherapists. According to the opinions of the three expert clinicians (pediatric rehabilitation doctor, pediatric psychiatrist, and pediatric neurologist), the clinical diagnosis was confirmed based on clinical manifestations and a

Table 1. Demographic characteristics of the study group $(\mathrm{n}=145)$

\begin{tabular}{|cc|}
\hline Characteristic & Value \\
\hline Age (mo) & $39.1 \pm 16.4$ \\
\hline $4-6$ & 8 \\
\hline $9-12$ & 10 \\
\hline $18-24$ & 20 \\
\hline $30-36$ & 39 \\
$42-48$ & 38 \\
\hline $54-60$ & 24 \\
\hline $66-71$ & 6 \\
\hline Sex & $100(69)$ \\
\hline Male & $45(31)$ \\
\hline Female & \\
\hline Disease group & 40 \\
\hline ASD & 46 \\
\hline GDD/ID & 37 \\
\hline DLS & \\
\hline
\end{tabular}

Values are presented as mean \pm standard deviation or number (\%).

ASD, autistic spectrum disorder; GDD/ID, global developmental delay/intellectual disability; DLD, developmental language disorder. 
battery of neuropsychiatric tests. As specific disabilities show delays only in specific areas [9], the participants were largely categorized into ASD, global developmental delay/intellectual disability (GDD/ID), and developmental language disorder (DLD) groups.

K-DST is composed of six domains (gross motor, fine motor, cognition, language, sociality, and self-care), and each domain consists of eight questions. The selfcare category is only applicable to participants aged 18 months or older. Each question was scored on a scale of 0-3 and each domain had a maximum attainable score of 24 points. Based on the previously analyzed standard deviation scores, the scores above 1 standard deviation (SD) were defined as 'advanced development', between -1 and 1 SD as 'appropriate for age', between -2 and - 1 SD as 'follow-up needed, and below -2 SD as 'further testing needed.' In this study, scores below -2 SD were classified as 'abnormal'.

K-ASQ is composed of five domains (communication, gross motor, fine motor, problem-solving, and personalsocial), and each domain included six questions. For each question, the answer of 'yes' was equivalent to 10 points, 'sometimes' was 5 points, and 'no' was 0 points. The maximum attainable score for each domain is 60 points. Unlike K-DST, whose results are subdivided into several categories according to variation from the norm, the K-ASQ scores below a determined cutoff are consid- ered 'abnormal'. If participants scored 'abnormal' values in at least one domain of each screening test, they were considered to have failed each test.

The study was approved by the Institutional Review Board of National Health Insurance Service Ilsan Hospital (No. 2017-04-037).

\section{Statistical analysis}

Based on the final diagnosis made by expert clinicians, sensitivity, specificity, positive predictive value, negative predictive value, and accuracy of diagnosis of developmental delay in each screening tool were analyzed.

The K-DST and K-ASQ scores were converted to percentages, and correlated with K-BSID-II and K-WPPSI-III scores using Pearson correlation to determine the developmental status based on the two screening tests. In the K-BSID, the mental and psychomotor development quotients were determined via correlation of the values obtained by dividing the calculated mental and psychomotor age by the actual age and multiplying by 100 . As many children had unspecified scores under ' 50 ', their mental and psychomotor development indices were not available. Moreover, in the K-WPPSI-III, the total, verbal, and performance intelligence quotients were used for correlation. The SPSS version 20.0 (IBM, Armonk, NY, USA) was used for all analysis.

Table 2. Sensitivity, specificity, and accuracy of K-ASQ and K-DST

\begin{tabular}{|c|c|c|c|c|c|}
\hline & \multicolumn{2}{|c|}{ K-DST } & \multicolumn{2}{|c|}{ K-ASQ } & \multirow{2}{*}{ p-value ${ }^{a}$} \\
\hline & Abnormal & Normal & Abnormal & Normal & \\
\hline Disease group $(\mathrm{n}=123)$ & 102 & 21 & 103 & 20 & \\
\hline $\operatorname{ASD}(n=40)$ & 38 & 2 & 35 & 5 & \\
\hline GDD/ID $(n=46)$ & 41 & 5 & 41 & 5 & \\
\hline $\operatorname{DLD}(\mathrm{n}=37)$ & 23 & 14 & 27 & 10 & \\
\hline Typically developing group $(\mathrm{n}=22)$ & 2 & 20 & 5 & 17 & \\
\hline Sensitivity (\%) & \multicolumn{2}{|c|}{$82.9(76.3-89.6)$} & \multicolumn{2}{|c|}{$83.7(77.2-90.3)$} & 0.7629 \\
\hline Specificity (\%) & \multicolumn{2}{|c|}{$90.9(78.9-100.0)$} & \multicolumn{2}{|c|}{$77.3(59.8-94.8)$} & 0.1615 \\
\hline $\operatorname{PPV}(\%)$ & \multicolumn{2}{|c|}{$98.1(95.4-100.0)$} & \multicolumn{2}{|c|}{$95.4(91.4-99.3)$} & 0.1804 \\
\hline NPV (\%) & \multicolumn{2}{|c|}{$48.8(33.5-64.1)$} & \multicolumn{2}{|c|}{$45.9(29.9-62.0)$} & 0.5734 \\
\hline Accuracy (\%) & \multicolumn{2}{|c|}{$84.1(78.2-90.1)$} & \multicolumn{2}{|c|}{$82.8(76.6-88.9)$} & 0.6168 \\
\hline
\end{tabular}

Values are presented as number or $95 \%$ confidence interval.

K-DST, Korean Developmental Screening Test; K-ASQ, Korean Ages and Stages Questionnaire; ASD, autistic spectrum disorder; GDD/ID, global developmental delay/intellectual disability; DLD, developmental language disorder; PPV, positive predictive value; NPV, negative predictive value.

${ }^{a}$ Between K-DST and K-ASQ. 


\section{RESULTS}

\section{Patient characteristics}

A total of 145 children (including 100 males) participated in this study. The participants' mean age was $39.1 \pm 16.4$ months. Developmental delay was finally diagnosed in 123 children, who included 40 diagnosed with ASD, 46 with GDD/ID, and another 37 with DLD (Table 1). Fifty-five participants were tested using K-BSID-II and 38 using K-WPPSI-III.
Sensitivities, specificities, and accuracies of K-DST and K-ASO based on final clinical diagnosis

The sensitivity and specificity of K-DST were $82.9 \%$ and $90.9 \%$, respectively. The sensitivity and specificity of K-ASQ based on final clinical diagnosis were $83.7 \%$ and $77.3 \%$, respectively. In the group with disorders, 102 participants were classified as abnormal in K-DST while 103 were classified as abnormal in K-ASQ. In the typically developing group, 20 participants were normal in K-DST and 17 were normal in K-ASQ. No statistical difference between K-DST and K-ASQ was found in terms of sensitivities, specificities, positive predictive values, negative

Table 3. Correlation between K-DST and K-BSID-II/K-WPPSI-III (Pearson correlation)

\begin{tabular}{|c|c|c|c|c|c|c|c|c|c|c|}
\hline \multirow{3}{*}{$\begin{array}{c}\text { Domains of } \\
\text { K-DST }\end{array}$} & \multicolumn{4}{|c|}{ K-BSID-II (n=55) } & \multicolumn{6}{|c|}{ K-WPPSI-III (n=38) } \\
\hline & \multicolumn{2}{|c|}{ MDQ } & \multicolumn{2}{|c|}{ PDQ } & \multicolumn{2}{|c|}{ TIQ } & \multicolumn{2}{|c|}{ VIQ } & \multicolumn{2}{|c|}{ PIQ } \\
\hline & $\mathbf{r}$ & p-value & $\mathbf{r}$ & p-value & $\mathbf{r}$ & p-value & $\mathbf{r}$ & p-value & $\mathbf{r}$ & p-value \\
\hline Gross motor & 0.440 & 0.001 & 0.605 & $<0.001$ & 0.083 & 0.618 & 0.010 & 0.954 & 0.217 & 0.191 \\
\hline Fine motor & 0.478 & $<0.001$ & 0.423 & 0.001 & 0.608 & $<0.001$ & 0.578 & $<0.001$ & 0.601 & $<0.001$ \\
\hline Cognition & 0.401 & 0.002 & 0.284 & 0.036 & 0.658 & $<0.001$ & 0.642 & $<0.001$ & 0.604 & $<0.001$ \\
\hline Language & 0.466 & $<0.001$ & 0.261 & 0.055 & 0.589 & $<0.001$ & 0.628 & $<0.001$ & 0.498 & 0.001 \\
\hline Sociality & 0.626 & $<0.001$ & 0.488 & $<0.001$ & 0.292 & 0.076 & 0.332 & 0.042 & 0.286 & 0.082 \\
\hline Self-care & 0.336 & 0.014 & 0.352 & 0.010 & 0.216 & 0.193 & 0.132 & 0.429 & 0.341 & 0.036 \\
\hline $\begin{array}{l}\text { Numbers of } \\
\text { abnormalities }\end{array}$ & -0.656 & $<0.001$ & -0.601 & $<0.001$ & -0.479 & 0.002 & -0.491 & 0.002 & -0.461 & 0.004 \\
\hline
\end{tabular}

K-DST, Korean Developmental Screening Test; K-BSID-II, Korean version of Bayley Scales of Infant Development-II; MDQ, metal development quotient; PDQ, psychomotor development quotient; K-WPPSI-III, Korean-Wechsler Preschool and Primary Scale of Intelligence-III; TIQ, total intelligence quotient; VIQ, verbal intelligence quotient; PIQ, performance intelligence quotient.

Table 4. Correlation between K-ASQ and K-BSID-II/K-WPPSI-III (Pearson correlation)

\begin{tabular}{|c|c|c|c|c|c|c|c|c|c|c|}
\hline \multirow{3}{*}{$\begin{array}{c}\text { Domains of } \\
\text { K-ASQ }\end{array}$} & \multicolumn{4}{|c|}{ K-BSID-II $(n=55)$} & \multicolumn{6}{|c|}{ K-WPPSI-III (n=38) } \\
\hline & \multicolumn{2}{|c|}{ MDQ } & \multicolumn{2}{|c|}{ PDQ } & \multicolumn{2}{|c|}{ TIQ } & \multicolumn{2}{|c|}{ VIQ } & \multicolumn{2}{|c|}{ PIQ } \\
\hline & $\mathbf{r}$ & p-value & $\mathbf{r}$ & p-value & $\mathbf{r}$ & p-value & $\mathbf{r}$ & p-value & $\mathbf{r}$ & p-value \\
\hline Gross motor & 0.398 & 0.003 & 0.538 & $<0.001$ & 0.031 & 0.852 & -0.045 & 0.789 & 0.117 & 0.483 \\
\hline Fine motor & 0.459 & $<0.001$ & 0.524 & $<0.001$ & 0.413 & 0.010 & 0.422 & 0.008 & 0.427 & 0.007 \\
\hline Problem solving & 0.649 & $<0.001$ & 0.493 & $<0.001$ & 0.433 & 0.007 & 0.484 & 0.002 & 0.410 & 0.011 \\
\hline Communication & 0.435 & 0.001 & 0.277 & 0.041 & 0.421 & 0.008 & 0.449 & 0.005 & 0.379 & 0.019 \\
\hline Personal-social & 0.551 & $<0.001$ & 0.467 & $<0.001$ & 0.175 & 0.294 & 0.193 & 0.246 & 0.217 & 0.190 \\
\hline $\begin{array}{l}\text { Numbers of } \\
\text { abnormalities }\end{array}$ & -0.685 & $<0.001$ & -0.687 & $<0.001$ & -0.351 & 0.031 & -0.437 & 0.006 & -0.311 & 0.058 \\
\hline
\end{tabular}

K-ASQ, Korean Ages and Stages Questionnaire; K-BSID-II, Korean version of Bayley Scales of Infant Development-II; MDQ, metal development quotient; PDQ, psychomotor development quotient; K-WPPSI-III, Korean-Wechsler Preschool and Primary Scale of Intelligence-III; TIQ, total intelligence quotient; VIQ, verbal intelligence quotient; PIQ, performance intelligence quotient. 
predictive values, or accuracies (Table 2).

\section{Correlation between screening tools and K-BSID-II and K-WPPSI-III}

Generally, both K-DST and K-ASQ showed moderate positive correlation with K-BSID-II (Tables 3, 4). The two screening tools also had higher correlation coefficients in K-WPPSI-III than in K-BSID-II. In terms of gross motor dysfunctions, both K-DST and K-ASQ showed a moderate positive correlation with K-BSID-II; however, no correlation was observed between total intelligence quotient and verbal intelligence quotient of K-WPPSI-III. The sociability domain in K-DST and the personal-social domains of K-ASQ also showed a weak correlation with KWPPSI-III. The self-care domain of K-DST showed a weak correlation with K-WPPSI-III.

\section{Comparison between K-DST and K-ASO}

On average, K-DST and K-ASQ showed a positive correlation with both K-BSID-II and K-WPPSI-III. The value of correlation coefficient between K-ASQ and K-BSID-II was higher than that of K-BSID-II with K-DST. However, the findings were not statistically significant (Table 5 ).

\section{DISCUSSION}

Although several screening tools were developed to evaluate developmental disability, they were not appropriate for use in actual clinical settings. DDST-II requires a long time to administer, and is hard to administer to all infants and children in the Korean medical environment. Additionally, as DDST-II is not based on a scoring system, it has poor selection accuracy [10]. K-ASQ, which was a substitute for DDST-II, was widely used abroad prior to the introduction of K-DST. K-ASQ was developed based on ASQ-II, which was designed in the United States in 1980, and was revised for use in Korea. K-ASQ encompasses 30 simple questions to be answered by the caregivers [10]. Unlike DDST-II that requires experts to administer, K-ASQ can be easily used by anyone in a relatively short time. Furthermore, it is useful for follow-ups in different age ranges. However, it was disputed whether the screening tool developed in the United States was reliable for use in infants or children growing up in a different cultural environment. Other studies have reported large discrepancies in reliabilities under different age ranges and the inaccurate definition of 'problem-solving' and 'personal-social' [11-13].

K-DST encompasses the health examination age range (4-71 months) and evaluates six areas of development (gross motor, fine motor, cognition, language, sociality, and self-care). Compared with DDST-II and K-ASQ, it is more segmented and interprets the results at four different levels (advanced development, age-appropriateness, need for follow-up, and need for further testing). Compared with prior screening tools, K-DST facilitates a more comprehensive developmental evaluation [6].

To our knowledge, this is the first study to directly compare K-DST and K-ASQ. One of the biggest differences between K-DST and K-ASQ was the fact that K-ASQ showed results as 'normal' and 'abnormal' while K-DST defined below -2 SD as 'abnormal' and scores between -1 SD and -2 SD as 'borderline' for later follow-up. In the K-DST, if the cutoff was adjusted from -2 SD to -1 SD, additional 29 pediatric cases were included under the 'fail' group. In the disorder group, only two of the 123 participants passed the K-DST. However, in the typically developing group, 12 subjects failed the K-DST. Additionally,

Table 5. Correlation between K-DST/K-ASQ average and K-BSID-II/K-WPPSI-III (Pearson correlation)

\begin{tabular}{|c|c|c|c|c|c|c|c|c|c|c|}
\hline & \multicolumn{4}{|c|}{ K-BSID-II $(n=55)$} & \multicolumn{6}{|c|}{ K-WPPSI-III (n=38) } \\
\hline & \multicolumn{2}{|c|}{ MDQ } & \multicolumn{2}{|c|}{ PDQ } & \multicolumn{2}{|c|}{ TIQ } & \multicolumn{2}{|c|}{ VIQ } & \multicolumn{2}{|c|}{ PIQ } \\
\hline & $\mathbf{r}$ & p-value & $\mathbf{r}$ & p-value & $\mathbf{r}$ & p-value & $\mathbf{r}$ & p-value & $\mathbf{r}$ & p-value \\
\hline Average of K-DST & $0.576^{* * *}$ & 0.8344 & $0.515^{* * *}$ & 0.9662 & $0.5879^{* *}$ & 0.7441 & $0.5665^{* *}$ & 0.8687 & $0.5959 * * *$ & 0.7788 \\
\hline Average of K-ASQ & $0.617^{* * *}$ & & $0.575^{* * *}$ & & $0.4586^{*}$ & & $0.4703^{*}$ & & $0.4749 *$ & \\
\hline
\end{tabular}

K-DST, Korean Developmental Screening Test; K-ASQ, Korean Ages and Stages Questionnaire; K-BSID-II, Korean version of Bayley Scales of Infant Development-II; MDQ, metal development quotient; PDQ, psychomotor development quotient; K-WPPSI-III, Korean-Wechsler Preschool and Primary Scale of Intelligence-III; TIQ, total intelligence quotient; VIQ, verbal intelligence quotient; PIQ, performance intelligence quotient. ${ }^{*} \mathrm{p}<0.05,{ }^{* *} \mathrm{p}<0.001,{ }^{* * *} \mathrm{p}<0.0001$ (comparison K-DST and K-ASQ). 
with similar adjustment in SD, the sensitivity increased from $82.9 \%$ to $98.4 \%$. However, the specificity was $45.5 \%$, which was significantly lower than the previous $90.9 \%$ when the cutoff was -2 SD. Specificity indicates the likelihood of correctly excluding disease in the typically developing individual; a low specificity increases the medical cost as it exposes normal individuals to unnecessary testing. Participants scoring from -1 SD to -2 SD on K-DST were further investigated.

When participants suspected of developmental disability were confirmed using K-DST and K-ASQ, no significant differences in sensitivities and specificities were observed. Moreover, both scales showed a high correlation with the standardized reference scales, K-BSID-II and KWPPSI-III. Additionally, both K-DST and K-ASQ showed high sensitivity and specificity, which indicated their acceptability as screening tools for the evaluation of developmental delay. Based on a comprehensive diagnosis, in the DLD group, the sensitivity of the screening tools was relatively low compared with ASD and GDD/ID. In patients with DLD, only two passed the K-DST when the cutoff expanded to -1 SD, while 14 passed the K-DST with $-2 \mathrm{SD}$ as the cutoff. Among these additional 12 participants, 7 were in the borderline category in the language domain of K-DST. Among 13 participants with borderline criteria in the language domain and none below -2 SD in all other domains, only 2 were diagnosed as normal (4 participants had GDD/ID and 7 had DLD). In the authors' experience and opinion, K-DST reflects the status of language development relatively well and DLD can be easily detected when the cutoff was expanded to -1 SD.

Interestingly, in terms of motor skills, the group with delayed gross motor skills showed a high proportion of abnormalities whereas the group with delayed fine motor skills had a low proportion of abnormalities. This observation may be explained by the gross motor skills, which developed before fine motor skills. As fine motor skills take longer to mature and are relatively more difficult to acquire than gross motor skills, it is possible that accurate measurements were not obtained in this study. Hence, borderline participants are more likely to be considered as normal in the future. However, the borderline group only included 29 participants who met the criteria. Hence, further investigation is necessary.

The strength of this study is that experienced experts provided accurate clinical diagnoses. However, this study had several limitations. First, the sample included a limited number of children. Second, the size of the typically developing group was especially smaller than the group with disorders, which might suggest limited generalizability. Furthermore, the age distribution was not uniform across each delayed disorder because of the small sample size. Therefore, a further large-scale study is needed to address these limitations.

In conclusion, K-DST is a reliable screening tool with mutually correlated results compared with K-BSID-II and K-WPPSI-III. Therefore, the K-DST may be used to replace K-ASQ.

\section{CONFLICT OF INTEREST}

No potential conflict of interest relevant to this article was reported.

\section{AUTHOR CONTRIBUTION}

Conceptualization: Kim SW. Methodology: Jeon HR, J Kim. Formal analysis: Jang CH. Funding acquisition: none. Project administration: DW Jung. Visualization: Lee JW. Writing - original draft: Jang CH. Writing - review and editing: Lee JW, Cho HE. Approval of final manuscript: all authors

\section{REFERENCES}

1. Rydz D, Shevell MI, Majnemer A, Oskoui M. Developmental screening. J Child Neurol 2005;20:4-21.

2. Anderson LM, Shinn C, Fullilove MT, Scrimshaw SC, Fielding JE, Normand J, et al. The effectiveness of early childhood development programs: a systematic review. Am J Prev Med 2003;24(3 Suppl):32-46.

3. Edwards SL, Sarwark JF. Infant and child motor development. Clin Orthop Relat Res 2005;(434):33-9.

4. Moon JS, Lee SY, Eun BL, Kim YK, Kim SW, Shin $\mathrm{SM}$, et al. One-year evaluation of the national health screening program for infants and children in Korea. Korean J Pediatr 2010;53:307-13.

5. Kim YJ, Lee JY, Sohn JA, Lee EH, Lee JA, Choi CW, et al. A validity study of the Korean Ages and Stages Questionnaires: screening for developmental delay in preterm infant. J Korean Soc Neonatol 2010;17:217-23.

6. Eun BL, Chung HJ, Shin SM, Oh KJ, Kim YA, Kim CT, 
et al. A study on the development and validation of Korean Developmental Screening Test for infants and children [Internet]. Osong: Korea Centers for Disease Control and Prevention; 2014 [cited 2019 Jul 1]. Available from: http://www.nih.go.kr/board.es?mid=a408 01000000\&bid=0050\&act=view\&list_no $=61795$.

7. Suh CR, Sohn SY, Kim GH, Jung SK, Eun BL. Singlecenter experience of the Korean-Developmental Screening Test for infants and children. Korean J Pediatr 2016;59:483-9.

8. Yim CH, Kim GH, Eun BL. Usefulness of the Korean Developmental Screening Test for infants and children for the evaluation of developmental delay in Korean infants and children: a single-center study. Korean J Pediatr 2017;60:312-9.

9. Blondis TA, Roizen NJ, Snow JH, Accardo PJ. Developmental disabilities: a continuum. Clin Pediatr (Phila) 1993;32:492-8.

10. Ga HY, Kwon JY. A comparison of the Korean-Ages and
Stages Questionnaires and Denver Developmental Delay Screening Test. Ann Rehabil Med 2011;35:36974.

11. Kim SW, Kim JY, Lee SY, Jeon HR. The comparison of M-B CDI-K Short Form and K-ASQ as screening test for language development. Ann Rehabil Med 2016; 40:1108-13.

12. Glascoe FP. Screening for developmental and behavioral problems. Ment Retard Dev Disabil Res Rev 2005;11:173-9.

13. Council on Children With Disabilities; Section on Developmental Behavioral Pediatrics; Bright Futures Steering Committee; Medical Home Initiatives for Children With Special Needs Project Advisory Committee. Identifying infants and young children with developmental disorders in the medical home: an algorithm for developmental surveillance and screening. Pediatrics 2006;118:405-20. 